\title{
Optimal Myocardial Boundary Estimation for MR Cardio Perfusion Measurements Using Sensitivity Analysis
}

\author{
LJ Spreeuwers $^{1}$, F Wierda ${ }^{1}$, M Breeuwer ${ }^{2}$ \\ ${ }^{1}$ Image Sciences Institute, University Medical Center Utrecht, Netherlands, \\ ${ }^{2}$ Medical Imaging Information Technology, Philips Medical Systems, Best, Netherlands
}

\begin{abstract}
Recent advances in Magnetic Resonance Imaging allow fast recording of contrast enhanced myocardial perfusion scans. MR perfusion scans are made by recording, during a period of 20-40 seconds a number of short-axis slices through the myocardium. For perfusion analysis, the myocardial boundaries must be traced, dividing the heart into the left and right ventricle blood volumes and the myocardium of the left ventricle. Extreme care must be taken not to include any part of the left or right ventricle blood volumes in the myocardium segment, because this has a significant effect on the perfusion analysis. On the other hand, for accurate estimation of the perfusion parameters, all available myocardium area in the image is required. In this paper a method is proposed to correct for any inclusion of left and right ventricle and optimally place the myocardial contours.
\end{abstract}

\section{Introduction}

\subsection{MR Cardio perfusion scans}

The perfusion of the myocardium can be measured using contrast enhanced Magnetic Resonance cardio perfusion scans. These scans are made by recording a number of short-axis slices through the heart (see fig.1), during a

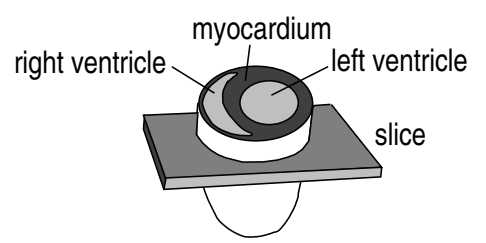

Figure 1. Short-axis slice through the heart

period of 20-40 seconds. The scanning is triggered by the patient's ECG, typically resulting in one set of slices per heart beat and each set of slices showing exactly the same phase in the contraction cycle of the heart. A contrast agent is injected in the patient and subsequently the heart is imaged. The contrast agent appears brightly in the MR images. In an image sequence, one can observe the contrast first entering the right ventricle, then the left ventricle and finally the perfusion in the myocardium (see fig.2).

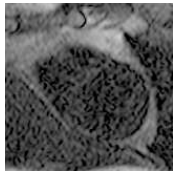

a

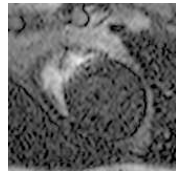

b

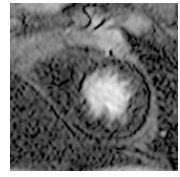

c

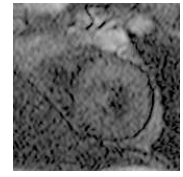

d
Figure 2. Four images of an MR cardio perfusion scan. a) before contrast; b) contrast in right ventricle; c) contrast in left ventricle; d) perfusion in myocardium

\subsection{Perfusion analysis}

Recent research has shown a good correlation between MR myocardial perfusion measurements and the presence of a coronary-artery disease [1, 2]. As a measure for perfusion in the myocardium, often the maximum upslope of the intensity-time curve of the myocardium is used. The intensity-time curve is the intensity of a position in the myocardium (or in the left or right ventricle) plotted as a function of time. Intensity-time curves for left and right ventricles and for the myocardium are shown in fig.3.

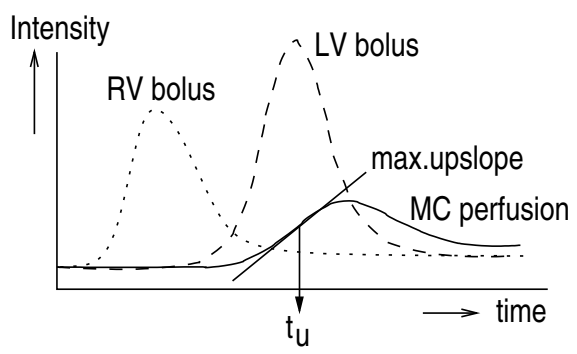

Figure 3. Intensity-time curves of left and right ventricles and myocardium. $t_{u}$ is the maximum upslope time

Since the signal in the myocardium is very noisy, generally the myocardium is divided into segments and the measurements are averaged over these segments [1, 3, 4]. 
These segments (see fig.4) coincide with areas of the myocardium which are supplied with blood from a certain coronary artery. In this way, if a reduced perfusion is observed in a myocardial segment, it can be traced back to the supplying artery.

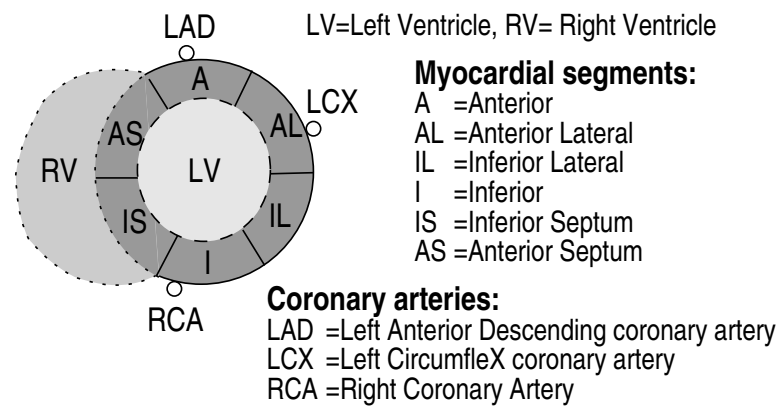

Figure 4. Division of the myocardium into 6 segments.

\subsection{Myocardial boundary requirements}

Since the signal strengths in the left and right ventricles are much higher than in the myocardium, extreme care should be taken not to include any part of the left and right ventricles into the myocardial segments. The boundaries of the left and ventricle blood volumes can be very irregular due to the presence of papillary muscles and trabeculae. Also the boundaries are often not clearly visible in a single image of the sequence or even on a maximum intensity projection through time. Furthermore, the images have a rather low resolution (typically $128 * 128$ pixels) and only few pixels are available for averaging within a segment. Currently, in most MR cardio perfusion analysis research, the myocardial boundaries are traced manually $[1,2,3,4]$. An automatic method was proposed in [5]. Fig.5 shows manually and automatically traced contours. As can be

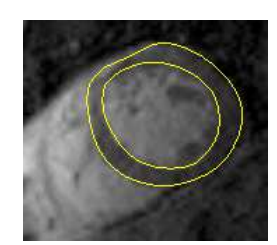

a

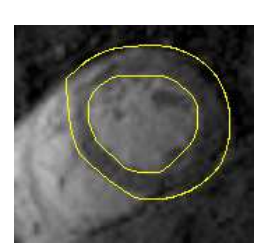

b
Figure 5. a) manually and b) automatically traced myocardial boundaries

observed, the myocardium can be rather thin. To obtain a good estimate for the maximum upslope, averaging must be done over as many pixels as is possible while at the same time inclusion of the left and right ventricle blood volumes must be avoided.

In literature on MR cardiac perfusion analysis, the effect of inclusion of the left and right ventricle blood volumes is hardly addressed at all. In the remainder of this paper this effect is analysed and a method for correction of the myocardial boundaries is presented.

\section{Contour correction}

In order to investigate the influence of the position of the myocardial boundaries on the estimated maximum upslope, we decided to calculate the sensitivity of the maximum upslope for the position of the boundaries. For each myocardial segment the inner boundary was moved over small distances relative to the centre of the left ventricle and for each position the average of the maximum upslope for the segment was calculated. This is illustrated in fig.6.

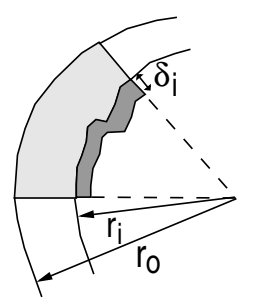

Original segment

Additional area due to boundary displacement

Figure 6. Displacement of inner boundary over distance $\delta_{i}$

Since the maximum upslope in the left ventricle is much higher than in the myocardium, one would expect the average maximum upslope in a segment to increase if more of the left ventricle is included. If no pixels of the left ventricle are included into the segment, the average maximum upslope should remain constant. For the time $t_{u}$ on which the maximum upslope takes place exactly the opposite behaviour can be expected. Since in the left ventricle $t_{u}$ is earlier than in the myocardium, including pixels of the left ventricle reduces the average of $t_{u}$ in the segment. For circle segments, the relation between the average maximum upslope $\bar{u}$ and and the average maximum upslope time $\bar{t}_{u}$ and the displacement $\delta_{i}$ can easily be derived analytically:

$$
\begin{gathered}
\bar{u}\left(\delta_{i}\right)=\frac{u_{m} A_{m}\left(\delta_{i}\right)+u_{l v} A_{l v}\left(\delta_{i}\right)}{A_{m}\left(\delta_{i}\right)+A_{l v}\left(\delta_{i}\right)} \\
\bar{t}_{u}\left(\delta_{i}\right)=\frac{t_{m} A_{m}\left(\delta_{i}\right)+t_{l v} A_{l v}\left(\delta_{i}\right)}{A_{m}\left(\delta_{i}\right)+A_{l v}\left(\delta_{i}\right)} \\
A_{m}\left(\delta_{i}\right)= \begin{cases}\delta_{i}<0 & \frac{\phi}{2}\left(r_{o}^{2}-r_{i}^{2}\right) \\
\delta_{i} \geq 0 & \frac{\phi}{2}\left(r_{o}^{2}-\left(r_{i}+\delta_{i}\right)^{2}\right)\end{cases} \\
A_{l v}\left(\delta_{i}\right)= \begin{cases}\delta_{i}<0 & \frac{\phi}{2}\left(r_{i}^{2}-\left(r_{i}+\delta_{i}\right)^{2}\right) \\
\delta_{i} \geq 0 & 0\end{cases}
\end{gathered}
$$

where $r_{i}$ and $r_{o}$ are the radii of the inner and outer boundaries, $u_{m}$ and $u_{l v}$ are the maximum upslope in the myocardium and the left ventricle $\left(u_{l v}>u_{m}\right), \phi$ is the 
angle of the segment ( $\phi=\frac{\pi}{3}$ if there are 6 segments), $A_{m}$ and $A_{l v}$ are the areas of the segment in the myocardium and the left ventricle and $\delta_{i}$ is the displacement, which is positive for outward and negative for inward displacements. In (2), $t_{m}$ is the maximum upslope time in the myocardium and $t_{l v}$ in the left ventricle $\left(t_{l v}<t_{m}\right)$. For these equations we assume that $u_{l v}, u_{m}, t_{l v}$ and $t_{m}$ are constant over the whole of the left ventricle and the myocardium. The curves in fig.7 were generated using these formulas and typical values for $u_{l v}, u_{m}, t_{l v}, t_{m}, r_{i}$ and $r_{o}$. From these curves it
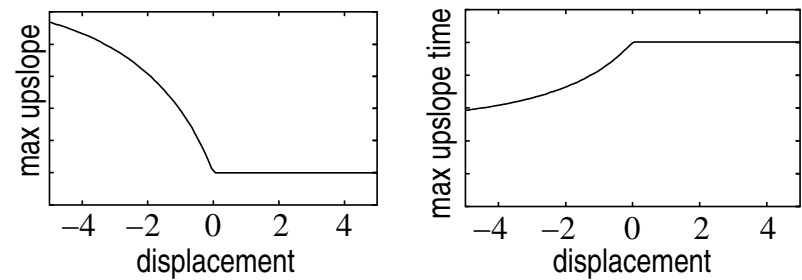

Figure 7. Max. upslope and upslope time $t_{u}$ as function of the displacement of the inner myocardial boundary

becomes clear that the optimal position of the boundary can be determined by finding the position where the maximum upslope and maximum upslope time remain constant when the boundary is moved outwards. If the displacement of the outer boundary is also taken into account, the equations become more complicated. For example for the two segments bordering on the right ventricle (IS and AS):

$$
\begin{gathered}
\bar{u}\left(\delta_{i}, \delta_{o}\right)=\frac{u_{r v} A_{r v}+u_{m} A_{m}+u_{l v} A_{l v}}{A_{r v}+A_{m}+A_{l v}} \\
\bar{t}_{u}\left(\delta_{i}, \delta_{o}\right)=\frac{t_{r v} A_{r v}+t_{m} A_{m}+t_{l v} A_{l v}}{A_{r v}+A_{m}+A_{l v}}
\end{gathered}
$$

$$
\begin{aligned}
& A_{r v}=\left\{\begin{array}{l}
\delta_{o}<0 \quad 0 \\
\delta_{o} \geq 0 \quad \frac{\phi}{2}\left(\left(r_{o}+\delta_{o}\right)^{2}-r_{o}^{2}\right)
\end{array}\right. \\
& A_{m}= \begin{cases}\delta_{o}>0 \wedge \delta_{i}<0 & \frac{\phi}{2}\left(r_{o}^{2}-r_{i}^{2}\right) \\
\delta_{o} \leq 0 \wedge \delta_{i}<0 & \frac{\phi}{2}\left(\left(r_{o}+\delta_{o}\right)^{2}-r_{i}^{2}\right) \\
\delta_{o}>0 \wedge \delta_{i} \geq 0 & \frac{\phi}{2}\left(r_{o}^{2}-\left(r_{i}+\delta_{i}\right)^{2}\right) \\
\delta_{o} \leq 0 \wedge \delta_{i} \geq 0 & \frac{\phi}{2}\left(\left(r_{o}+\delta_{o}\right)^{2}-\left(r_{i}+\delta_{i}\right)^{2}\right)\end{cases} \\
& A_{l v}= \begin{cases}\delta_{i}<0 & \frac{\phi}{2}\left(r_{i}^{2}-\left(r_{i}+\delta_{i}\right)^{2}\right) \\
\delta_{i} \geq 0 & 0\end{cases}
\end{aligned}
$$

Where $A_{r v}$ is the area of the segment in the right ventricle, $u_{r v}$ and $t_{r v}$ are the maximum upslope and maximum upslope time in the right ventricle and $\delta_{o}$ is the displacement of the outer boundary. In fig. 8 an example is given of the resulting behaviour of the maximum upslope and maximum upslope time for a small positive $\delta_{o}$ as a function of $\delta_{i}$.
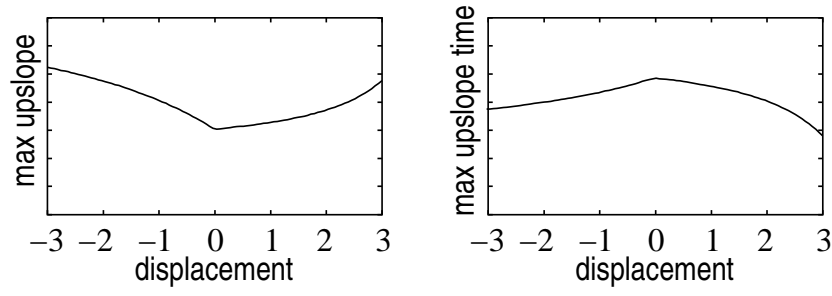

Figure 8. Max. upslope and upslope time $t_{u}$ as function of the displacement of the inner myocardial boundary with a small outward displacement of the outer boundary

\section{Experiments and results}

For a set of 9 image sequences, the myocardial boundaries were manually traced and 6 segments were assigned as in figure 4 . The boundaries were displaced over a range of \pm 3 pixels by sub-sampling the curve with a one degree step and moving each point relative to the centre of gravity of the boundary over a distance defined by the displacement. For each pixel the maximum upslope was calculated by fitting a piecewise linear function to the intensity-time profile and finding the position and steepness of the centre of the slope. An example of the found sensitivity curves is shown in fig.9. These are the
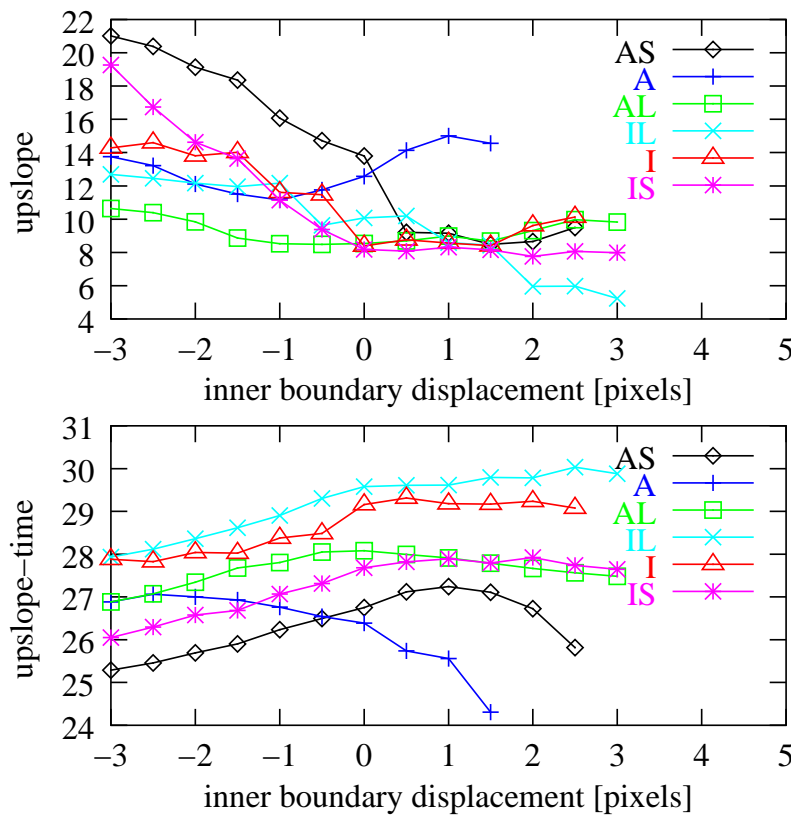

Figure 9. Max. upslope and upslope time $t_{u}$ as function of the displacement of the inner myocardial boundary

curves for the manual segmentation of fig.5a. As expected, the position of the myocardial boundaries indeed has a large influence on the resulting maximum upslope and maximum upslope time. E.g. moving the inner boundary 
of the $A S$ segment outward half a pixel reduces the average maximum upslope from 14 to 9 . The results in fig.9 show good correspondence with the predicted behaviour in fig.7 (the $A L, I L, I$ and $I S$ segments) and fig.8 (the $A S$ and $A$ segments). Due to the presence of the papillars (where the upslope is lower), the increase of the upslope in the $A, A L$, $I L$ and $I$ segments is less than the increase in the $A S$ and $I S$ segments if the inner-boundary is moved inwards, The $A$ segment borders on a tail of the right ventricle and part of the right ventricle is included. This is why the $A$ segment shows the behaviour of fig.8. A similar analysis can be performed for the outer myocardial boundary.

In tables 1 and 2 the found inner and outer contour displacements and uncorrected and corrected average maximum upslope and maximum upslope time for the contours in figure 5 are given.

\begin{tabular}{|l|r|r|r|r|r|r|}
\hline segment & $\bar{u}$ & $\bar{u}_{t}$ & $\delta_{i}$ & $\delta_{o}$ & $\bar{u}$-corr & $\bar{u}_{t}$-corr \\
\hline AS & 13.8 & 26.8 & 0.5 & -1.0 & 8.9 & 28.5 \\
A & 12.6 & 26.4 & -1.0 & -1.5 & 7.9 & 28.2 \\
L & 8.5 & 28.0 & -0.5 & -0.5 & 8.8 & 28.2 \\
IL & 10.0 & 29.6 & 0.0 & 0.0 & 10.0 & 29.6 \\
I & 8.4 & 29.2 & 0.0 & 0.0 & 8.4 & 29.2 \\
IS & 8.2 & 27.7 & 0.0 & 0.0 & 8.2 & 27.7 \\
\hline
\end{tabular}

Table 1. Displacements and uncorrected and corrected values of the upslope and upslope time for the manually traced contours in fig.5a

\begin{tabular}{|l|r|r|r|r|r|r|}
\hline segment & $\bar{u}$ & $\bar{u}_{t}$ & $\delta_{i}$ & $\delta_{o}$ & $\bar{u}$-corr & $\bar{u}_{t}$-corr \\
\hline AS & 11.8 & 25.5 & 0.0 & -2.0 & 9.5 & 28.3 \\
A & 24.4 & 24.9 & 1.0 & -3.0 & 11.4 & 27.6 \\
L & 10.5 & 27.7 & 0.0 & -2.0 & 7.7 & 28.2 \\
IL & 12.3 & 28.8 & 1.0 & -0.5 & 9.9 & 29.3 \\
I & 13.6 & 28.5 & 0.5 & -1.0 & 11.1 & 29.2 \\
IS & 9.2 & 27.3 & 0.5 & 0.0 & 8.7 & 27.6 \\
\hline
\end{tabular}

Table 2. Displacements and uncorrected and corrected values of the upslope and upslope time for the automatically traced contours in fig. $5 \mathrm{~b}$

The corrected values allow much more consistent comparison between the segments and different boundary tracing methods. Fig.10 shows a comparison between

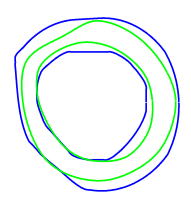

a

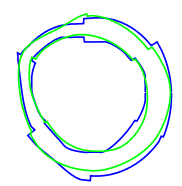

b
Figure 10. Comparison of a) uncorrected and b) corrected manually and automatically traced boundaries

the manually and automatically traced boundaries of fig.5 before and after the boundary position correction. After the correction the boundaries are much closer to each-other.

\section{Conclusions}

A method is proposed for analysis of the effect of the position of myocardial boundaries on myocardial perfusion analysis using contrast enhanced MR perfusion scans. The analysis shows that the position of the myocardial boundaries is very important for accurate perfusion analysis. The sensitivity of perfusion parameters for these boundary positions corresponds well with the predictions of a simplified model, which is described in this paper as well. Using these sensitivity measurements, a correction for the boundary positions can be determined which results in more reliable and consistent perfusion analysis.

\section{References}

[1] Wilke N, Jerosch-Herold M, Wang Y, Hunag Y, Christensen $\mathrm{B}$, Stillman A, Ugurbil $\mathrm{K}$, McDonald $\mathrm{K}$, Wilson $\mathrm{R}$. Myocardial perfusion reserve: assessment with multisection, quantitative, fi rst-pass MR imaging. Radiology 1997; 204(2):373-384.

[2] Al-Saadi N, Nagel E, Gross M, Bornstedt A, Schnackenburg $\mathrm{B}$, Klein $\mathrm{C}$, Oswald $\mathrm{H}$, Fleck E. Noninvasive detection of myocardial ischemia from perfusion reserve based on cardiovascular magnetic resonance. Circulation 2000; 101:1379-1383.

[3] Schwitter J, von Schulthess G. Echo-planar MR perfusion imaging is highly reliable in detection of cor onary-artery disease. In Proceedings Int. Society of Magnetic Resonance In Medicine. Denver, Colorado, USA, 1-7 April 2000; 34.

[4] Schmitta M, Schreiber W, Petersen S, Kreitner K, Scharhag J, Klessen C, Voigtländer T, Meyer J, Thelen M. Quantifi cation of myocardial perfusion and perfusion reserve with magnetic fi rst-pass measurements on patients with known coronary artery disease. In Proceedings Int. Society of Magnetic Resonance In Medicine. Denver, Colorado, USA, 1-7 April 2000; 531.

[5] Spreeuwers L, Breeuwer M. Automatic detection of the myocardial boundaries of the right and left ventricle in MR cardio perfusion scans. In Proceedings SPIE Medical Imaging, volume 4322. San Diego, USA, 19-22 Febr. 2001; 1207-1217.

Address for correspondence:

Luuk Spreeuwers

Image Sciences Institute

University Medical Center

Heidelberglaan 100, 3584 CX Utrecht, Netherlands

tel./fax: +31-30-250-6682/+31-30-251-3399

luuk@isi.uu.nl 